VOL. I (1969), 195-208.

\title{
On regular semigroups whose idempotents form a subsemigroup
}

\author{
T. E. Hall
}

\begin{abstract}
For brevity the semigroups in the title are called orthodox semigroups. The finest inverse semigroup congruence on an orthodox semigroup is shown to have a simple form and conversely, regular semigroups whose finest inverse congruence has this simple form are shown to be orthodox. Next ideal extensions of orthodox semigroups by orthodox semigroups are shown to be also orthodox, whence a finite semigroup is orthodox if and only if each principal factor is orthodox and completely o-simple or simple. Finally it is determined which completely 0-simple semigroups are orthodox.
\end{abstract}

\section{Introduction and summary}

An orthodox semigroup is a regular semigroup in which the set of idempotents forms a subsemigroup. Results concerning orthodox semigroups have been given by Fantham [2], Howie and Lallement [3], Meakin [6], Reilly and Scheiblich [7], and Yamada [9]. Lemma 1.3 [7] shows that orthodox semigroups are precisely those regular semigroups with the property that $b^{\prime} a^{\prime}$ is an inverse of $a b$ whenever $a^{\prime}, b^{\prime}$ are inverses of $a, b$ respectively.

For each element $a$ of an orthodox semigroup $S$ define $V(a)=\{x \in S: a x a=a$ and $x a x=x\}$, the set of inverses of $a$. We consider (in Section 4) the relation $y=\{(x, y) \in S \times S: V(x)=V(y)\}$ and

Received 21 March 1969. Received by J. Austral. Math. Soc. 30 October 1968. Communicated by G.B. Preston. This research was carried out at Monash University while the author held a Commonwealth Postgraduate Award. 
show that it is the finest inverse congruence on $S$. The natural converse is also proved. In [9], Yamada has used this congruence on any generalized inverse semigroup (a regular semigroup in which efgh = egfh, for any idempotents $e, f, g, h)$ to determine generalized inverse semigroups in terms of inverse semigroups.

The proofs of the above results of section 4 are made easy by the main result of section 3, namely that a regular semigroup $S$ is orthodox if and only if, for any elements $a, b$ in $S, V(a) \cap V(b) \neq \square$ implies $V(a)=V(b)$.

It is well known that if $I$ is an ideal of a semigroup $S$, then $I$ and $S / I$ are regular [inverse] if and only if $S$ is regular [inverse]. The bracketed result is Corollary 7.37 [1], volume 2 p. 58. In Section 5 we prove the corresponding result for orthodox semigroups (Theorem 4). Theorem 5 and Examples 1 and 2 deal with the sixteen similar statements in which the two sets of the idempotents of the regular semigroups $S / I$ and $I$ each satisfy any one of the following four permutation identities: [left][right] normality and commutativity (for definitions, see Section 2).

A corollary to Theorem 4 is that a finite semigroup is orthodox if and only if each of its principal factors is an orthodox completely O-simple semigroup or an orthodox completely simple semigroup. In Section 6 we determine which completely o-simple semigroups are orthodox.

\section{Preliminaries}

We use whenever possible the notation of Clifford and Preston [1]. We also use the following three results.

RESULT 1. (from Lemma 1.3, Reilly and Scheiblich [7]) For any regular semigroup $S$, the following three conditions are equivalent.

(i) $S$ is orthodox.

(ii) For any elements $a, b$ in $S$ and any elements $a^{\prime}$ in $V(a)$ and $b^{\prime}$ in $V(b)$, the element $b^{\prime} a^{\prime}$ is an inverse of $a b$.

(iii) If $e$ is any idempotent of $S$ and $x \in V(e)$, then $x$ is also an idempotent.

RESULT 2. (from Lemma 2.2, Lallement [5]) Let $\rho$ be a congruence on a regular semigroup $S$. Then each $p$-class which is a subsemigroup 
contains an idempotent.

From this we easily obtain,

RESULT 3. On a regular semigroup $S$ define

$$
\sigma=\left\{(e f, f e) \in S \times S: e^{2}=e \text { and } f^{2}=f\right\} .
$$

Then $\sigma^{*}$, the congruence generated by $\sigma$, is the finest inverse congruence on $S$. Moreover, if $\rho$ is any congmence on $S$ which contains $\sigma^{*}$, then $S / \rho$ is also inverse.

The latter statement follows from the fact that any homomorphic image of an inverse semigroup is also an inverse semigroup (Theorem 7.36, [1], volume 2 p. 57 ).

We give now some results due to Kimura and Yamada, (see Section 5 of [4] and Section 4 of [9]). Let $X=\left\{x_{1}, x_{2}, \ldots, x_{n}\right\}$ be a set in which each element $x_{i}$ is called a variable. By a permutation identity in the variables $x_{1}, x_{2}, \ldots, x_{n}$ we mean a pair of words $\left(x_{1} x_{2} \ldots x_{n}, x_{p_{1}} x_{p_{2}} \ldots x_{p_{n}}\right.$ where $\left(p_{1}, p_{2}, \ldots, p_{n}\right)$ is a nontrivial permutation of $(1,2, \ldots, n)$. The permutation identity above is commonly written as $x_{1} x_{2} \ldots x_{n}=x_{p_{1}} x_{p_{2}} \ldots x_{p_{n}}$. The following is a list of some permutation identities and their common nomes:

$$
\begin{aligned}
& x_{1} x_{2}=x_{2} x_{1}, \quad \text { commutativity; } \\
& x_{1} x_{2} x_{3}=x_{1} x_{3} x_{2}, \text { left normality; } \\
& x_{1} x_{2} x_{3}=x_{2} x_{1} x_{3}, \text { right normality; }
\end{aligned}
$$

and

$$
x_{1} x_{2} x_{3} x_{4}=x_{1} x_{3} x_{2} x_{4}, \text { normality. }
$$

The set of idempotents $B$ of a regular semigroup $S$ is said to satisfy the permutation identity $x_{1} x_{2} \ldots x_{n}=x_{p_{1}} x_{p_{2}} \cdots x_{p_{n}}$, if, for each mapping $\phi$ from $X$ into $B$, we have

$$
\phi\left(x_{1}\right) \phi\left(x_{2}\right) \ldots \phi\left(x_{n}\right)=\phi\left(x_{p_{1}}\right) \phi\left(x_{p_{2}}\right) \ldots \phi\left(x_{p_{n}}\right) .
$$

From Lemma 3 of [9], if the set of idempotents of a regular semigroup $S$ satisfies a permutation identity, then $S$ is orthodox. Two permutation 
identities are equivalent if, for each regular semigroup $S$, the set of idempotents of $S$ satisfies both the permutation identities or neither. We can now state the following result (see, for example, Section 4 [9], p. 389). The permutation identity $x_{1} x_{2} \ldots x_{n}=x_{p_{1}} x_{p_{2}} \ldots x_{p_{n}}$ is equivalent to
(i) commutativity if $p_{1} \neq 1$ and $p_{n} \neq n$
(ii) left normality if $p_{1}=1$ and $p_{n} \neq n$
(iii) right normality if $p_{1} \neq 1$ and $p_{n}=n$
(iv) normality if $p_{1}=1$ and $p_{n}=n$.

\section{3. $V(a)$}

Let $S$ be an orthodox semigroup and let $B$ be its set of idempotents. From Exercise 1, $\$ 4.2$, [1], volume 1, p. 129, the band $B$ is a semilattice of rectangular bands. We may assume then that $B=\underset{\alpha \in Y}{\bigcup_{\alpha}}$ where

(i) each $E_{\alpha}$ is a rectangular band,

(ii) $Y$ is a semilattice, and for all $\alpha, \beta \in Y$, we have

(iii) $E_{\alpha} \cap E_{\beta}=\square$ if $\alpha \neq \beta$ and

(iv) $E_{\alpha \beta} E_{\beta} \subseteq E_{\alpha \beta}$.

If $e \in E_{\alpha}$ then we shall sometimes denote $E_{\alpha}$ by $E(e)$. Then for any elements $e, f \in B$, we have $E(e) E(f) \subseteq E(e f)=E(f e)$, and from result 1 it easily follows that $V(e)=E(e)$.

LEMMA 1 For each element $a \in S$ there exist elements $\alpha, \beta \in Y$ such that $a V(a) \subseteq E_{\alpha}$ and $V(a) a \subseteq E_{\beta}$.

Proof. Take any element $a^{\prime} \in V(a)$. Then of course there exist elements $\alpha, \beta \in Y$ such that $a a^{\prime} \in E_{\alpha}$ and $a^{\prime} a \in E_{\beta}$. Take any other element $a^{*} \in V(a)$. Then

$$
\left(a a^{\prime}\right)\left(a a^{*}\right)=\left(a a^{\prime} a\right) a^{*}=a a^{*} \text { and }\left(a a^{*}\right)\left(a a^{\prime}\right)=a a^{\prime} \text {. }
$$

It follows that $E\left(\alpha \alpha^{*}\right)=E\left(\alpha \alpha^{\prime}\right)=E_{\alpha}$, whence $\alpha a^{*} \in E_{\alpha}$ and so $a V(a) \subseteq E_{\alpha}$. Dually, we infer that $V(a) a \subseteq E_{\beta}$. 
DEFINITION 1. For each pair $\alpha, \beta \in Y$, define

$$
S_{\alpha, \beta}=\left\{x \in S: x V(x) \subseteq E_{\alpha} \text { and } V(x) x \subseteq E_{\beta}\right\} \text {. }
$$

From Lemma 1, the semigroup $S$ is the disjoint union of the sets $\left\{S_{\alpha, \beta}: \alpha, \beta \in Y\right\}$. Note that in general it is not true that each set $S_{\alpha, \beta}$ is non-void. (In fact $S_{\alpha, \beta} \neq \square$ if and only if $E_{\alpha}$ and $E_{\beta}$ are contained in the same $D$-class of $S$. )

LEMMA 2. For any elements $\alpha, \beta \in Y$, if $a \in S_{\alpha, \beta}$ then $V(\alpha) \subseteq S_{\beta, \alpha}$.

Proof. We merely have to note that, from lemma 1, it follows that $S_{\alpha, \beta}=\left\{x \in S:\right.$ for some $x^{\prime} \in V(x), x x^{\prime} \in E_{\alpha}$ and $\left.x^{\prime} x \in E_{\beta}\right\}$.

THEOREM 1. For any elements $\alpha, \beta \in Y$ and $a \in S$ and any element $a^{\prime} \in V(a)$, if $a \in S_{\alpha, \beta}$, then

$$
V(a)=E_{\beta} a^{\prime} E_{\alpha}
$$

Proof. (i) Take any element $a^{*} \in V(a)$. Then $a^{*}=a^{*} a a^{*}=a^{*}\left(a a^{\prime} a\right) a^{*}=\left(a^{*} a\right) a^{\prime}\left(a a^{*}\right) \in E_{\beta} a^{\prime} E_{\alpha}$.

Hence $\quad V(a) \subseteq E_{B} a^{\prime} E_{\alpha}$.

(ii) Take any elements $f \in E_{B}$ and $e \in E_{\alpha}$. Then

$$
\begin{aligned}
a\left(f a^{\prime} e\right) a & =\left(a a^{\prime} a\right) f\left(a^{\prime} a a^{\prime} a a^{\prime}\right) e\left(a a^{\prime} a\right) \\
& =a\left[\left(a^{\prime} a\right) f\left(a^{\prime} a\right)\right] a^{\prime}\left[\left(a a^{\prime}\right) e\left(a a^{\prime}\right)\right] a \\
& =a\left[\left(a^{\prime} a\right)\left(a^{\prime} a\right)\right] a^{\prime}\left[\left(a a^{\prime}\right)\left(a a^{\prime}\right)\right] a \\
& =a \\
\left(f a^{\prime} e\right) a\left(f a^{\prime} e\right) & =f\left(a^{\prime} a a^{\prime}\right) e\left(a a^{\prime} a a^{\prime} a\right) f\left(a^{\prime} a a^{\prime}\right) e \\
& =f a^{\prime}\left[\left(a a^{\prime}\right) e\left(a a^{\prime}\right)\right] a\left[\left(a^{\prime} a\right) f\left(a^{\prime} a\right)\right] a^{\prime} e \\
& =f a^{\prime}\left[\left(a a^{\prime}\right)\left(a a^{\prime}\right)\right] a\left[\left(a^{\prime} a\right)\left(a^{\prime} a\right)\right] a^{\prime} e \\
& =f a^{\prime} e .
\end{aligned}
$$

Hence $f a^{\prime} e \in V(a)$ and thus $E_{\beta} a^{\prime} E_{\alpha} \subseteq V(a)$.

THEOREM 2. A regular semigroup $S$ is orthodox if and only if, for any elements $a, b$ in $S, V(a) \cap V(b) \neq \square$ implies $V(a)=V(b)$. In fact a regular semigroup $S$ is orthodox if for any idempotents $e, f$ in $S, V(e) \cap V(f) \neq \square$ implies $V(e)=V(f)$. 
Proof. (i) Suppose $S$ is orthodox, and let us adopt the notation used above. Suppose $x \in V(a) \cap V(b)$ and that $x \in S_{B, \alpha}$ say. From Lemma 2 we have that $a, b \in V(x) \subseteq S_{\alpha, \beta}$ and from Theorem $I$ it follows that

$$
V(a)=E_{\beta} x E_{\alpha}=V(b)
$$

(ii) Suppose $S$ is a regular semigroup such that, for any idempotents $e, f$ in $S, V(e) \cap V(f) \neq \square$ implies $V(e)=V(f)$. Let $g$ be any idempotent of $S$ and take any element $x \in V(g)$. Then of course $x g$ and $g x$ are idempotents and one readily obtains that $g \in V(x g) \cap V(g x)$. Hence

$$
x g \in V(x g)=V(g x)
$$

and so

$$
(x g)(g x)(x g)=x g
$$

Therefore

$$
(x g)(g x)(x g) x=x g x
$$

and so

$$
x^{2}=x
$$

That is, $x$ is an idempotent. From Result 1, it follows that $S$ is orthodox.

\section{The finest inverse congruence}

THEOREM 3. If $S$ is any orthodox semigroup, then the relation $y=\{(x, y) \in S \times S: V(x)=V(y)\}$ is the finest inverse congmience on $S$. Conversely, if $S$ is a regular semigroup and the relation $y=\{(x, y) \in S \times S: V(x)=V(y)\}$ is an inverse congmence on $S$, then $S$ is an orthodox semigroup.

Proof. (i) Suppose $S$ is orthodox. The relation $y$ is clearly an equivalence relation. Take any pair $(a, b) \in Y$ and any element $c$ in $S$. Take any elements $x \in V(a) \cap V(b)$ and $c^{\prime} \in V(c)$. From Result 1, we have that $c^{\prime} x \in V(a c) \cap V(b c)$ and $x c^{\prime} \in V(c a) \cap V(c b)$. Hence $V(a c)=V(b c)$ and $V(c a)=V(c b)$ and so $(a c, b c)$ and $(c a, c b)$ are contained in $y$. Therefore $y$ is compatible.

To show that $S / Y$ is an inverse semigroup, consider any two 
idempotents $e, f$ in $S$. Then $V(e f)=E(e f)=E(f e)=V(f e)$ and so $(e f, f e) \in Y$. Thus the relation $\sigma=\left\{(e f, f e) \in S \times S: e^{2}=e, f^{2}=f\right\}$ is contained in $y$ and so $\sigma^{*}$, the congruence generated by $\sigma$, is also contained in $y$. From result $3, S / Y$ is inverse.

We have denoted the finest inverse congruence by $\sigma^{*}$. Take any pair $(a, b)$ in $y$. Then $V(a)=V(b)$. Choose any element $x$ from $V(a)$. Then $a \sigma^{*}$ and $b \sigma^{*}$ are inverses of $x \sigma^{*}$ in $S / \sigma^{*}$, an inverse semigroup, and so $a \sigma^{*}=b \sigma^{*}$. That is, $(a, b) \in \sigma^{*}$ and so $y \subseteq \sigma^{*}$. It follows that $y$ is the finest inverse congruence on $S$.

(ii) Suppose $S$ is a regular semigroup and that $y$ is an inverse congruence on $S$. Take any elements $a, b$ in $S$ for which $V(a) \cap V(b) \neq \square$. Take an element $x$ in $V(a) \cap V(b)$. Then $a y$ and $b y$ are both inverses of $x y$ in $S / y$, an inverse semigroup. Therefore $a y=b y$, whence $(a, b) \in Y$ and $V(a)=V(b)$. From theorem $2, S$ is orthodox.

REMARK 1. From theorem 2, it can easily be shown that $a Y=V\left(a^{\prime}\right)$, for any element $a$ in an orthodox semigroup $S$ and any element $a^{\prime}$ in $V(a)$. For $x \in V\left(a^{\prime}\right)$ if and only if $V(x)=V(a)$, and so

$$
\begin{aligned}
a^{Y} & =\{x \in S: V(x)=V(a)\} \\
& =\left\{x \in S: x \in V\left(a^{\prime}\right)\right\} \\
& =V\left(a^{\prime}\right) .
\end{aligned}
$$

Moreover, if $e$ is any idempotent of $S$, then

$$
e^{y}=V(e)=E(e) \text {. }
$$

\section{Ideal extensions}

THEOREM 4. Let $I$ be an ideal of a semigroup $S$. Then $S$ is orthodox if and only if $S / I$ and $I$ are orthodox.

Proof. (i) Suppose $S / I$ and $I$ are orthodox semigroups. Take any idempotent $e$ in $S$ and any element $x \in V(e)$. Then one readily obtains that $e$ and $x$ are contained in the same J-class of $S$ and both are contained in $S \backslash I$ or both are contained in $I$. From Result 1 , since $S / I$ and $I$ are both orthodox, it is clear that $x$ is an idempotent. Again from Result 1 , it follows that $S$ is orthodox. A rather similar proof uses Theorem 2 . 
(ii) The "only if" statement is obvious. In fact, from Result 2, it is obvious that any homomorphic image of an orthodox semigroup is also orthodox, and clearly any regular subsemigroup of an orthodox semigroup is also orthodox.

COROLLARY 1. (to the proof) A semigroup $S$ is orthodox if and only if each of its principal factors is orthodox.

COROLLARY 2. A finite semigroup is orthodox if and only if each of its principal factors is an orthodox completely 0 -simple semigroup, or an orthodox completely simple semigroup.

THEOREM 5. Let $I$ be an ideal of a semigroup $S$ and suppose $I$ is an inverse semigroup. Then $S$ is a regular semigroup whose idempotents satisfy a permutation identity if and only if $S / I$ is a regular semigroup whose idempotents satisfy the same permutation identity.

Proof. (i) Suppose $S / I$ is a regular semigroup whose idempotents satisfy a permutation identity. By Lemma 3 [9] (see Section 2) and Theorem $4, S$ is an orthodox semigroup. Its set of idempotents $B$ say, is a semilattice of rectangular bands. As in Section 3 , let $B=\bigcup_{\alpha \in Y} E_{\alpha}$ and for $e \in E_{\alpha}$ denote $E_{\alpha}$ by $E(e)$.

For each $\alpha \in Y, E_{\alpha} \subseteq S \backslash I$ or $E_{\alpha} \subseteq I$. (For suppose there is an element $f \in E_{\alpha}$ such that $f \in I$. Then $E_{\alpha}=E_{\alpha} f E_{\alpha} \subseteq I$.) Thus for any idempotent $e \in I$, we have that $E(e)=\{e\}$ (since $E(e)$ is a commutative rectangular band).

Let the permutation identity be $x_{1} x_{2} \ldots x_{n}=x_{p_{1}} x_{p_{2}} \ldots x_{p_{n}}$ and take any idempotents $e_{1}, e_{2}, \ldots, e_{n}$ belonging to $S$.

Case 1. Suppose $e_{1} e_{2} \ldots e_{n} \in S \backslash I$. Then $e_{p_{1}} e_{p_{2}} \cdots e_{p_{n}} \in E\left(e_{1} e_{2} \ldots e_{n}^{\prime} \subseteq S \backslash I\right.$ and clearly $e_{1} e_{2} \ldots e_{n}=e_{p_{1}} e_{p_{2}} \cdots e_{p_{n}}$.

Case 2. Suppose $e_{1} e_{2} \ldots e_{n} \in I$. Then

$$
e_{p_{1}} e_{p_{2}} \ldots e_{p_{n}} \in E\left(e_{1} e_{2} \ldots e_{n}\right)=\left\{e_{1} e_{2} \ldots e_{n}\right\}
$$


and so

$$
e_{1} e_{2} \ldots e_{n}=e_{p_{1}} e_{p_{2}} \cdots e_{p_{n}}
$$

Thus the set of idempotents of $S$ also satisfies the permutation identity $x_{1} x_{2} \ldots x_{n}=x_{p_{1}} x_{p_{2}} \cdots x_{p_{n}}$.

(ii) The "only if" statement is obvious. In fact, from Result 2 it is obvious that if $S$ is any regular semigroup whose idempotents satisfy a permutation identity, then any homomorphic image of $S$ is also a regular semigroup whose idempotents satisfy the same permutation identity.

The following examples show that no similar result holds if we assume only that $I$ is regular and its set of idempotents satisfies left normality, right normality or normality.

EXAMPLES 1 and 2. Let $I=\{a, b\}$ be a left zero semigroup and put $S=I^{1}$, the semigroup obtained from $I$ by adjoining an identity element. Then $S / I$ is a semilattice (and hence satisfies every permutation identity) and $I$ is a left normal (and hence also a normal) band, but $S$ satisfies no permutation identity, since it is not normal ( $1 a b 1=a$ and $1 b a 1=b$ ). The semigroups $S$ and $S^{*}$ (the semigroup dual to $S$ ) are the two orthodox semigroups of least order which are not generalized inverse semigroups.

\section{Completely 0-simple semigroups}

Our main aim in this section is to determine which completely 0-simple semigroups are orthodox. (Exercise $2(b)$ for $\$ 3.2$ of [1], volume 1, gives a determination of which completely simple semigroups are orthodox). Such a semigroup has of course a O-restricted inverse homomorphic image. ( $y$ of Theorem 3 is 0-restricted.) At first we shall assume only that our completely 0-simple semigroup has a 0-restricted inverse homomorphic image.

Let $S=S^{\circ}$ be a completely 0-simple semigroup. Because of Theorem 3.5 [1] volume 1, p. 94 , we may assume that $S=M^{\circ}(G ; I, \Lambda ; P)$, the Rees $I \times \Lambda$ matrix semigroup over a group with zero $G^{\circ}$, and with sandwich matrix $P=\left(p_{\lambda i}\right)$. Let the identity element of $G$ be $e$.

LEMMA 3. If the completely o-simple semigroup $S=M^{\circ}(G ; I, \Lambda ; P)$ has a o-restricted inverse homomorphic image then for some index set $J$, 
there exist partitions $P(I)=\left\{I_{j}: j \in J\right\}$ and $P(\Lambda)=\left\{\Lambda_{j}: j \in J\right\}$ of $I$ and $\Lambda$ respectively, such that, for any elements $i \in I$ and $\lambda \in \Lambda$,

$$
p_{\lambda i}=0 \text { if } i \in I_{j} \text { and } \lambda \in \Lambda_{k} \text { say, and } j \neq k
$$

and

$$
p_{\lambda i} \neq 0 \text { if } i \in I_{j} \text { and } \lambda \in \Lambda_{k} \text { say, and } j=k \text {. }
$$

Proof. Let $\Theta$ be a O-restricted homomorphism such that $S \Theta$ is an inverse semigroup ( $S \Theta$ is of course a Brandt semigroup) and, for some index set $J$, Iet $\left\{e_{j}: j \in J\right\}$ be the set of distinct, non-zero idempotents of $S \Theta$. For each $j \in J$, define

$$
\begin{aligned}
& I_{j}=\left\{i \in I: \text { for some } \lambda \in \Lambda, p_{\lambda i} \neq 0 \text { and }\left(p_{\lambda i}^{-1}\right)_{i \lambda} \Theta=e_{j}\right\}, \\
& \Lambda_{j}=\left\{\lambda \in \Lambda: \text { for some } i \in I, p_{\lambda i} \neq 0 \text { and }\left(p_{\lambda i}^{-1}\right)_{i \lambda} \Theta=e_{j}\right\} .
\end{aligned}
$$

Take any element $i \in I$ and any elements $\lambda, \mu \in \Lambda$ such that $p_{\lambda i} \neq 0$ and $p_{\mu i} \neq 0$. Then

$$
\left(p_{\lambda i}^{-1}\right)_{i \lambda}\left(p_{\mu i}^{-1}\right)_{i \mu}=\left(p_{\mu i}^{-1}\right)_{i \mu} \text { and }\left(p_{\mu i}^{-1}\right)_{i \mu}\left(p_{\lambda i}^{-1}\right)_{i \lambda}=\left(p_{\lambda i}^{-1}\right)_{i \lambda} \text {. }
$$

Since $S \Theta$ is an inverse semigroup it follows that $\left(p_{\lambda i}^{-1}\right)_{i \lambda} \Theta=\left(p_{\mu i}^{-1}\right)_{i \mu} \Theta$. Thus if $j \neq k$, we have $I_{j} \cap I_{k}=\square$. Hence $\left\{I_{j}: j \in J\right\}=P(I)$, say, is a partition of $I$ and similarly $\left\{\Lambda_{j}: j \in J\right\}=P(\Lambda)$, say, is a partition of $\Lambda$.

Now take any elements $j, k \in J$ and take any elements $i \in I_{j}$ and $\lambda \in \Lambda_{k}$. Then there exist elements $\mu \in \Lambda$ and $l \in I$ such that $p_{\mu i} \neq 0$ and $\left(p_{\mu i}^{-1}\right)_{i \mu} \Theta=e_{j}$, and $p_{\lambda l} \neq 0$ and $\left(p_{\lambda l}^{-1}\right){ }_{Z \lambda} \Theta=e_{k}$. It follows that

$$
\left[\left(p_{\lambda l}^{-1}\right)_{\tau_{\lambda}}\left(p_{\mu i}^{-1}\right)_{i \mu}\right] \Theta=e_{k} e_{j}
$$

Now if $j \neq k$, we have that $e_{k} e_{j}=0$ and, since $\theta$ is 0-restricted, that $p_{\lambda i}=0$. If $j=k$, then $e_{k} e_{j} \neq 0$ and $p_{\lambda i} \neq 0$. The statement of the lemma now follows. 
REMARK 2. Using Theorem 1 [8], we can also prove the converse. For suppose such partitions $P(I)$ and $P(\Lambda)$ exist and suppose $P$ is in normal form (see $\S 2[8]$ ). Let $N$ be the smallest normal subgroup containing the sets

$$
\left\{p_{\lambda i}^{-1} p_{\mu i}: i \in I, \lambda, \mu \in \Lambda \text { and } p_{\lambda i} \neq 0 \neq p_{\mu i}\right\}
$$

and

$$
\left\{p_{\lambda i}^{-1} p_{\lambda j}: i, j \in I, \lambda \in \Lambda \text { and } p_{\lambda i} \neq 0 \neq p_{\lambda j}\right\}
$$

or equivalently, the set $\left\{p_{\lambda i}: i \in I, \lambda \in \Lambda\right.$ and $\left.p_{\lambda i} \neq 0\right\}$. Then the congruence on $S$ given by the triple consisting of $P(I), P(\Lambda)$ and the congruence on $G$ induced by $N$, is O-restricted and is the finest inverse congruence on $S$.

For completeness, instead of applying the statement of Lemma 1 [8], we shall specialize its proof to treat the simple case in which the sandwich matrix $P$ has the diagonal block form described in Lemma 3 .

For each $j \in J$, choose a representative element $i_{j} \in I_{j}$ and a representative element $\lambda_{j} \in \Lambda_{j}$. Define a function $\phi$ from $I$ into $\Lambda$ as follows. For each element $i \in I$, put $i \phi=\lambda_{j}$, where $i \in I_{j}$. Define a function $\psi$ from $\Lambda$ into $I$ similarly by putting, for each element $\lambda \in \Lambda, \lambda \psi=i_{j}$, where $\lambda \in \Lambda_{j}$. Define $\Lambda \times \Lambda$ and $I \times I$ diagonal matrices $U=\left(u_{\lambda \mu}\right)$ and $V=\left(v_{i l}\right)$ respectively, as follows:

$$
\begin{aligned}
& u_{\lambda \mu}= \begin{cases}0 & \text { if } \lambda \neq \mu, \\
p_{\lambda, \lambda \psi}^{-1} & \text { if } \lambda=\mu,\end{cases} \\
& v_{i \tau}= \begin{cases}0 & \text { if } i \neq l, \\
p_{i \phi, i}^{-1} p_{i \phi, i \phi \psi} & \text { if } i=z .\end{cases}
\end{aligned}
$$

Set $U P V=P^{\prime}=\left(p_{\lambda i}^{\prime}\right)$, say. Then for each $i \in I$, 


$$
\begin{aligned}
p_{i \phi, i}^{\prime} & =u_{i \phi, i \phi} p_{i \phi, i} v_{i, i} \\
& =p_{i \phi, i \phi \psi}^{-1} p_{i \phi, i} p_{i \phi, i}^{-1} p_{i \phi, i \phi \psi} \\
& =e,
\end{aligned}
$$

and for each $\lambda \in \Lambda$,

$$
\begin{aligned}
p_{\lambda, \lambda \psi}^{\prime} & =u_{\lambda, \lambda} p_{\lambda, \lambda \psi} v_{\lambda \psi, \lambda \psi} \\
& =p_{\lambda, \lambda \psi}^{-1} p_{\lambda, \lambda \psi} p_{\lambda \psi \phi, \lambda \psi}^{-1} p_{\lambda \psi \phi, \lambda \psi \phi \psi} \\
& =p_{\lambda, \lambda \psi}^{-1} p_{\lambda, \lambda \psi} p_{\lambda \psi \phi, \lambda \psi}^{-1} p_{\lambda \psi \phi, \lambda \psi}
\end{aligned}
$$

since $\lambda \psi \phi \psi=i_{j}=\lambda \psi$, where $\lambda \in \Lambda_{j}$. Thus $p_{\lambda, \lambda \psi}^{\prime}=e$. More informally, each diagonal block of $P^{\prime}$ contains at least one row and one column in which each element is $e$. Let us now assume that $S=M^{0}(G ; I, \Lambda ; P)$ is orthodox and that we have obtained $P^{\prime}$ as above. Set $S^{\prime}=M^{0}\left(G ; I, \Lambda ; P^{\prime}\right)$. By Corollary 3.12 , [1] volume 1 p. 106, the semigroups $S$ and $S^{\prime}$ are isomorphic. Take any elements $i \in I$ and $\lambda \in \Lambda$ such that $p_{\lambda i}^{\prime} \neq 0$. Then since, for some $j \in J$, we have $\lambda \in \Lambda_{j}$ and $i \in I_{j}$, it follows that $i \phi \psi=i_{j}=\lambda \psi$. Hence

$$
p_{i \phi, i}^{\prime}=p_{\lambda, \lambda \psi}^{\prime}=p_{i \phi, i \phi \psi}^{\prime}=p_{i \phi, \lambda \psi}^{\prime}=e \text {. }
$$

Hence $(e)_{i, i \phi}$ and $(e)_{\lambda \psi_{,} \lambda}$ are idempotents of $S^{\prime}$ and their product $(e)_{i, i \phi}(e)_{\lambda \psi, \lambda}=(e)_{i \lambda}$ is also an idempotent. Thus $\left(p_{\lambda i}^{\prime-1}\right)_{i \lambda}=(e)_{i \lambda}$ and so $p_{\lambda i}^{\prime}=e$. The following theorem is now obvious.

THEOREM 6. The completely o-simple semigroup $S=M^{\circ}(G ; I, \Lambda ; P)$ is orthodox if and only if there exist diagonal $\Lambda \times \Lambda$ and $I \times I$ matrices $U$ and $V$ respectively, over $G^{\circ}$, such that the matrix $P^{\prime}=\left(p_{\lambda i}^{\prime}\right)=U P V$ has the following form (with a suitable ordering of $I$ and $\Lambda$ ): 


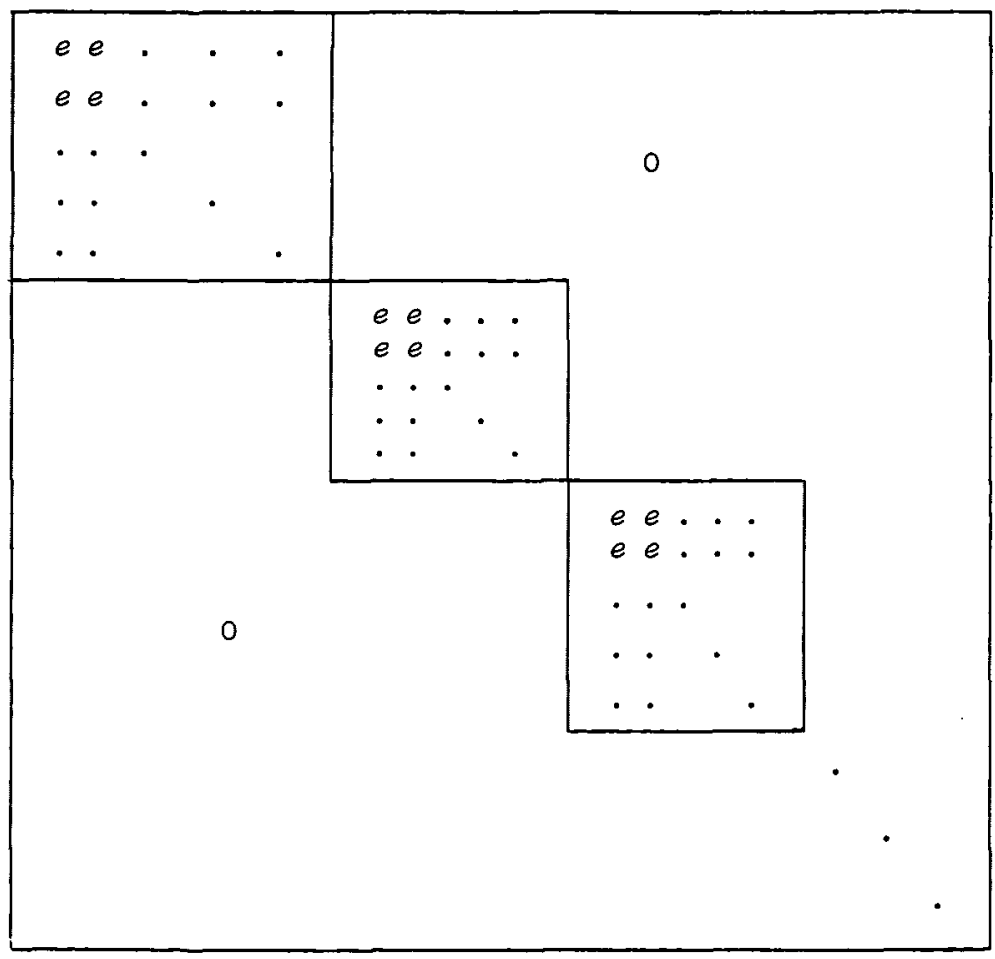

More formally, for some index set $J$, there exist partitions $P(I)=\left\{I_{j}: j \in J\right\}$ and $P(\Lambda)=\left\{\Lambda_{j}: j \in J\right\}$ of $I$ and $\Lambda$ respectively, such that

$$
p_{\lambda, i}^{\prime}=\left\{\begin{array}{l}
0 \text { if } i \in I_{j} \text { and } \lambda \in \Lambda_{k}, \text { say, and } j \neq k, \\
e \text { if } i \in I_{j} \text { and } \lambda \in \Lambda_{k}, \text { say, and } j=k .
\end{array}\right.
$$

\section{Acknowledgements}

The term "orthodox" is due to D.G. Fitzgerald and use of the symbol "y" was suggested by J.M. Howie. I am grateful to G.B. Preston for pointing out to me the two questions answered by Theorem 3 and for reading the first two drafts of this paper and suggesting many improvements. I am grateful to T. Saitô for also suggesting improvements. 


\section{References}

[1] A.H. Clifford and G.B. Preston, The algebraic theory of semigroups, vols. 1 and 2, (Amer. Math. Soc. Mathematical Surveys 7, Providence, 1961 and 1967).

[2] P.H.H. Fantham, "On the classification of a certain type of semigroup", Proc. London Math. Soc. (3) 10 (1960), 409-427.

[3] J.M. Howie and G. Lallement, "Certain fundamental congruences on a regular semigroup", Proc. Glasgow Math. Assoc. 7 (1965-66), 145-149.

[4] Naoki Kimura and Miyuki Yamada, "Note on idempotent semigroups II", Proc. Japan Acad., 34 (1958), 110-112.

[5] G. Lallement, "Congruences et équivalences de Green sur un demi-groupe régulier", C.R. Acad. Sci. Paris, Série A, 262 (1966), 613-616.

[6] J.C. Meakin, "Congruences on orthodox semigroups", J. Austral. Math. Soc. (to appear).

[7] N.R. Reilly and H.E. Scheiblich, "Congruences on regular semigroups", Pacific J. Math. 23 (1967), 349-360.

[8] Takayuki Tamura, "Decompositions of a completely simple semigroup", Osaka Math. J. 12 (1960), 269-275.

[9] Miyuki Yamada, "Regular semigroups whose idempotents satisfy permutation identities", Pacific J. Math. 21 (1967), 371-392.

Department of Mathematics, Monash University, Clayton, victoria. 